\title{
Imaging Responses to Immunotherapy with Novel PET Tracers
}

\author{
Anna-Larissa Niemeijer ${ }^{1}$, Otto S. Hoekstra ${ }^{2}$, Egbert F. Smit ${ }^{3}$, and Adrianus J. de Langen ${ }^{3}$ \\ ${ }^{1}$ Department of Pulmonary Diseases, Amsterdam UMC, Vrije Universiteit Amsterdam, Amsterdam, The Netherlands; ${ }^{2}$ Department of \\ Radiology and Nuclear Medicine, Amsterdam UMC, Vrije Universiteit Amsterdam, Amsterdam, The Netherlands; and ${ }^{3}$ Department of \\ Thoracic Oncology, Netherlands Cancer Institute, Amsterdam, The Netherlands
}

$\mathbf{I}$ cancers. Monoclonal antibodies (mAbs) targeting specific immune checkpoints, in particular the programmed-death 1 (PD-1), programmed death ligand 1 (PD-L1), and cytotoxic T-lymphocyteassociated antigen 4 (CTLA-4) receptors, are active against a wide range of solid and hematologic cancers. Durable responses that can last for years are now seen for disease settings that were treatmentrefractory before the era of immunotherapy. However, despite the success of immune checkpoint inhibitors (ICIs), only a subset of patients benefits, and to a variable extent. Primary as well as acquired resistance limit their efficacy. The underlying mechanisms of primary and acquired resistance are actively being analyzed in numerous trials designed to target these mechanisms. In melanoma, for example, the response rate to nivolumab (PD-1 mAb) and ipilimumab (CTLA-4 mAb) was 58\% (1) and the 5-y progression-free survival $36 \%$ (2). For most solid cancers, these numbers are even lower. Therefore, there is an urgent need for a priori discrimination of responders from nonresponders and for identification of those patients who will develop acquired resistance despite having experienced an initial good response to ICI therapy.

Biomarker development aims to select the optimal treatment strategy for the individual patient. Already during drug development, biomarkers can provide essential feedback on target inhibition and intended tumor microenvironmental changes that are associated with drug efficacy. The number of clinical trials being designed to evaluate the large number of possible ICI drug combinations exceeds the number of patients eligible for enrollment. Pharmacodynamic biomarkers can help speed the development of high-potential drugs and halt the development of less potent drugs at an early stage, thereby limiting the number of large-scale phase III studies with negative results.

In this Hot Topics article, we will discuss the opportunities for PET as an imaging biomarker in the field of ICI therapy.

\section{RESPONSE PREDICTION TO ICI THERAPY: PD-L1 EXPRESSION QUANTIFICATION}

The most widely used biomarker for patient selection in the clinical setting is PD-L1 immunohistochemistry. Patients with high expression of PD-L1 in the tumor membrane generally have a higher

Received Dec. 9, 2019; revision accepted Feb. 15, 2020.

For correspondence or reprints contact: Adrianus J. de Langen, Department of Thoracic Oncology, Netherlands Cancer Institute, Plesmanlaan 121, 1066 CX, Amsterdam, The Netherlands.

E-mail: j.d.langen@nki.nl

Published online Feb. 21, 2020.

COPYRIGHT @ 2020 by the Society of Nuclear Medicine and Molecular Imaging. DOI: 10.2967/jnumed.119.236158 chance of obtaining a response to PD-(L)1 mAb therapy. However, this biomarker is certainly not optimal. Non-small cell lung cancer patients whose tumor shows at least 50\% PD-L1 expression have a response rate of $45 \%$ to $\mathrm{PD}$-1-directed treatment (3), whereas patients without tumor PD-L1 expression still have a $10 \%$ chance of obtaining a response $(4,5)$. The limited positive predictive value of PD-L1 immunohistochemistry might be caused by treatment resistance mechanisms involving pathways beyond the PD-1-PD-L1 axis, such as insufficient tumor antigenicity, impaired antigen presentation, or an immunosuppressive microenvironment. The limited negative predictive value of PD-L1 immunohistochemistry might be caused by heterogeneity of PD-L1 expression within tumors $(6,7)$. Whole-body PET/CT with radiolabeled PD-L1 tracers enables the visualization and quantification of PD-L1 throughout the body, albeit at a lower resolution than immunohistochemistry performed on a tumor sample.

Three PD-L1 PET tracers have made it to the clinic so far. These studies show that PD-L1 tracer uptake is heterogeneous between patients, as well as within patients between different tumor lesions, and that tracer uptake correlates with tumor PD-L1 expression as measured by immunohistochemistry (8-10). Intriguingly, Bensch et al. reported that tracer uptake correlated with treatment response better than did PD-L1 immunohistochemistry (10).

Together, these studies show that whole-body PD-L1 PET/CT reveals, and can partially overcome, the limitations of PD-L1 quantification on a small biopsy. However, larger studies are required to validate the observations made in these pilot studies. Also, signal interpretation might differ between small-molecule and full $\mathrm{mAb}$ PD-L1 tracers because tracer uptake is a result of tumor perfusion, interstitial pressure, aspecific tissue distribution, and the nature of target binding (reversible vs. irreversible).

\section{CHALLENGES OF RESPONSE EVALUATION AND NEW, PROMISING PET TRACERS}

Response monitoring during ICI therapy is complex because of a phenomenon called pseudoprogression. As a result of a massive immune cell influx, tumors can initially grow as a result of treatment efficacy. Therefore size-based response criteria may underestimate treatment efficacy $(11,12)$. Receptor blockade by antagonistic mAbs of inhibitory immune checkpoint receptors such as PD-1 and CTLA4 , or receptor activation by agonistic mAbs of activating immune checkpoint receptors such as tumor necrosis factor receptor superfamily member $4(\mathrm{OX}-40)$ and glucocorticoid-induced tumor necrosis factor receptor, aim to activate CD8-positive T cells and shift the immune microenvironment in tumors from an immunosuppressive state to an immune-activated one. These biologic effects 
occur early during ICI therapy and precede volumetric changes. ${ }^{18} \mathrm{~F}-\mathrm{FDG}$, the most widely used tracer in the clinic, is hampered by its inability to discriminate between high metabolism of tumor cells and immune cells $(13,14)$. The early phase of an immune response is characterized by a mixture of tumor cell kill (i.e., decrease in ${ }^{18} \mathrm{~F}$-FDG accumulation) and activation and influx of immune cells (i.e., increase in ${ }^{18}$ F-FDG accumulation), and it is therefore unlikely that ${ }^{18} \mathrm{~F}$-FDG will be suitable for early response evaluation during ICI treatment. A different approach would be to monitor CD8-positive T-cell changes. Two T-cell PET tracers have entered the clinical arena so for. Pandit-Taskar et al. imaged 6 patients with solid malignancies using ${ }^{89} \mathrm{Zr}$-Df-IAB22M2C, an antiCD8 minibody, and PET/CT (15), whereas Colevas et al. imaged 5 patients with head and neck cancer in a study with ${ }^{18} \mathrm{~F}$-AraG PET/CT (16). This tracer is an ${ }^{18} \mathrm{~F}$-labeled analog of arabinofuranosyl guanine (AraG), a compound that shows selective accumulation in activated CD8-positive T cells. Preliminary results from both studies show that tracer injection is safe, and biodistribution suggests successful targeting of benign and malignant CD8-positive T-cell-rich tissues.

In contrast to early response evaluation, ${ }^{18} \mathrm{~F}-\mathrm{FDG}$ PET/CT seems to be an accurate biomarker to identify patients who are at risk of disease relapse despite having obtained an initial good response. In a retrospective analysis of 104 melanoma patients who were evaluated after 1 y of ICI treatment, only $28 \%$ had a complete response on CT whereas $75 \%$ had a complete metabolic response (17). In the group of patients who obtained a partial response on CT, ${ }^{18} \mathrm{~F}-\mathrm{FDG}$ PET/CT was able to identify patients with a high risk of disease progression: patients with residual ${ }^{18} \mathrm{~F}$-FDG uptake had a progression-free survival rate of $48 \%$, whereas those with a complete metabolic response had a progressionfree survival rate of $93 \%$. In other words, late-stage ${ }^{18} \mathrm{~F}-\mathrm{FDG}$ PET/ CT can be used to select patients for treatment intensification.

\section{OPPORTUNITIES FOR PET/CT TO AID ICI DRUG DEVELOPMENT}

PD-(L)1 and CTLA-4 checkpoint inhibitor therapy is able to induce long-term responses in a proportion of patients with late-stage treatment-refractory cancers such as melanoma and non-small cell lung cancer. The ultimate goal of engaging the immune system against tumors is to provide a cure for patients with cancers that are currently treatment-refractory. Our understanding of tumor biology, immune biology, and their interaction fuels therapeutic advances and drug development. PET/CT may facilitate this process. It has unique features that allow for noninvasive monitoring of virtually every biologic pathway in a whole-body fashion, and it can be repeated over time to monitor changes without influencing the tumor. By radiolabeling intact drugs in an inert way, one can visualize and quantify biodistribution. During drug combination therapy, drug-drug interactions that can lead to changes in drug delivery to the tumor or immune organs can be monitored (18).

ICI-ICI combination studies should be smart-designed. Timing of different immune modulators is critical to optimally stimulate immuneactivating cells and inhibit immune-suppressive cells. Messenheimer et al., for example, showed that sequential instead of concurrent OX-40 agonist and PD-1 antagonist treatment resulted in better immune cell activation against tumor cells (19). Treatment with the OX-40 agonist provided an initial boost in PD-1-positive CD4-positive and PD1-positive CD8-positive antitumor T cells that maximized after $13 \mathrm{~d}$ of OX-40 treatment and was extended only by subsequent treatment with a PD-1 mAb. Serial PET/CT would be an ideal way to monitor these changes and prevent the need for repeated biopsies.

\section{CONCLUSION}

PET/CT using novel immune tracers can help monitor and develop ICI therapy. The biomarker is in its early stage of development, in which validation and qualification are ongoing. Larger-scale molecular imaging studies with adequate data modeling and feedback from tissue analysis and outcome on treatment are required to evaluate the full potential of PET/CT in the field of ICI therapy.

\section{DISCLOSURE}

No potential conflict of interest relevant to this article was reported.

\section{REFERENCES}

1. Larkin J, Chiarion-Sileni V, Gonzalez R, et al. Combined nivolumab and ipilimumab or monotherapy in untreated melanoma. N Engl J Med. 2015;373:23-34.

2. Larkin J, Chiarion-Sileni V, Gonzalez R, et al. Five-year survival with combined nivolumab and ipilimumab in advanced melanoma. N Engl J Med. 2019;381: 1535-1546.

3. Reck M, Rodriguez-Abreu D, Robinson AG, et al. Pembrolizumab versus chemotherapy for PD-L1-positive non-small-cell lung cancer. N Engl J Med. 2016; 375:1823-1833.

4. Borghaei H, Paz-Ares L, Horn L, et al. Nivolumab versus docetaxel in advanced nonsquamous non-small-cell lung cancer. N Engl J Med. 2015;373:1627-1639.

5. Rittmeyer A, Barlesi F, Waterkamp D, et al. Atezolizumab versus docetaxel in patients with previously treated non-small-cell lung cancer (OAK): a phase 3 , open-label, multicentre randomised controlled trial. Lancet. 2017;389:255-265.

6. Li C, Huang C, Mok TS, et al. Comparison of 22C3 PD-L1 expression between surgically resected specimens and paired tissue microarrays in non-small cell lung cancer. J Thorac Oncol. 2017;12:1536-1543.

7. McLaughlin J, Han G, Schalper KA, et al. Quantitative assessment of the heterogeneity of PD-L1 expression in non-small-cell lung cancer. JAMA Oncol. 2016;2:46-54.

8. Niemeijer AN, Leung D, Huisman MC, et al. Whole body PD-1 and PD-L1 positron emission tomography in patients with non-small-cell lung cancer. Nat Commun. 2018;9:4664.

9. Xing Y, Chand G, Liu C, et al. Early phase I study of a ${ }^{99 \mathrm{~m}} \mathrm{Tc}-$ labeled anti-programmed death ligand-1 (PD-L1) single-domain antibody in SPECT/CT assessment of PD-L1 expression in non-small cell lung cancer. J Nucl Med. 2019;60:1213-1220.

10. Bensch F, van der Veen EL, Lub-de Hooge MN, et al. ${ }^{89} \mathrm{Zr}$-atezolizumab imaging as a non-invasive approach to assess clinical response to PD-L1 blockade in cancer. Nat Med. 2018;24:1852-1858.

11. Hodi FS, Hwu WJ, Kefford R, et al. Evaluation of immune-related response criteria and RECIST v1.1 in patients with advanced melanoma treated with pembrolizumab. J Clin Oncol. 2016;34:1510-1517.

12. Tazdait M, Mezquita L, Lahmar J, et al. Patterns of responses in metastatic NSCLC during PD-1 or PDL-1 inhibitor therapy: comparison of RECIST 1.1, irRECIST and iRECIST criteria. Eur J Cancer. 2018;88:38-47.

13. Humbert $\mathrm{O}$, Cadour N, Paquet M, et al. ${ }^{18}$ FDG PET/CT in the early assessment of non-small cell lung cancer response to immunotherapy: frequency and clinical significance of atypical evolutive patterns. Eur J Nucl Med Mol Imaging. November 23, 2019 [Epub ahead of print].

14. Rossi G, Bauckneht M, Genova C, et al. Comparison between ${ }^{18}$ F-FDG-PETand CT-based criteria in non-small cell lung cancer (NSCLC) patients treated with nivolumab. J Nucl Med. December 5, 2019 [Epub ahead of print].

15. Pandit-Taskar N, Postow M, Hellmann M, et al. First-in-human imaging with ${ }^{89} \mathrm{Zr}$-Df-IAB22M2C anti-CD8 minibody in patients with solid malignancies: preliminary pharmacokinetics, biodistribution, and lesion targeting. J Nucl Med. October 4, 2019 [Epub ahead of print].

16. Colevas AD, Bedi N, Chang S, et al. A study to evaluate immunological response to PD-1 inhibition in squamous cell carcinoma of the head and neck (SCCHN) using novel PET imaging with [18F]F-AraG [abstract]. J Clin Oncol. 2018;36:6050.

17. Tan AC, Emmett L, Lo S, et al. FDG-PET response and outcome from anti-PD-1 therapy in metastatic melanoma. Ann Oncol. 2018;29:2115-2120.

18. Van der Veldt AA, Lubberink M, Bahce I, et al. Rapid decrease in delivery of chemotherapy to tumors after anti-VEGF therapy: implications for scheduling of anti-angiogenic drugs. Cancer Cell. 2012;21:82-91.

19. Messenheimer DJ, Jensen SM, Afentoulis ME, et al. Timing of PD-1 blockade is critical to effective combination immunotherapy with anti-OX40. Clin Cancer Res. 2017;23:6165-6177. 